\title{
Dolutegravir - a review of the pharmacology, efficacy, and safety in the treatment of HIV
}

This article was published in the following Dove Press journal:

Drug Design, Development and Therapy

7 July 2015

Number of times this article has been viewed

\author{
Christopher E Kandel' \\ Sharon L Walmsley ${ }^{1,2}$ \\ 'Department of Medicine, University \\ of Toronto, Toronto, ON, Canada; \\ ${ }^{2}$ Division of Infectious Diseases, \\ University Health Network, Toronto, \\ ON, Canada
}

\begin{abstract}
Dolutegravir is the newest integrase strand transfer inhibitor to be approved for the treatment of human immunodeficiency virus (HIV) infection. Dolutegravir is equivalent or superior to existing treatment regimens in both treatment-naïve and treatment-experienced patients including those with previous raltegravir or elvitegravir failure. The consistent efficacy coupled with excellent tolerability and infrequent drug-drug interactions makes the co-formulation of dolutegravir with two nucleotide reverse-transcriptase inhibitors an attractive treatment option. This review summarizes the pharmacokinetics, adverse event profile, and efficacy of dolutegravir in the treatment of HIV.
\end{abstract}

Keywords: patient-reported outcomes, integrase inhibitor, antiretroviral therapy

\section{Introduction}

Since the approval of zidovudine in 1987, there has been an increasing number of antiretroviral agents developed targeting the human immunodeficiency virus (HIV). The current antiretroviral classes include the nucleoside and nucleotide reverse-transcriptase inhibitors (NRTIs), non-nucleoside reverse-transcriptase inhibitors (NNRTIs), protease inhibitors (PIs), entry inhibitors, and integrase strand transfer inhibitors (INSTIs). ${ }^{1,2}$ Increasingly, co-formulations are constructed with the goal of enhancing adherence, thereby ensuring the continual HIV suppression necessary to avoid the development of resistance. ${ }^{3}$ Antiretroviral therapy has lengthened the average life span of HIV-infected individuals to approach that of the general population while concurrently increasing the burden of comorbidities. ${ }^{4,5}$ Accordingly, there is an increasing need for agents with few drug-drug interactions, reduced toxicity, high genetic barrier to resistance, low pill burden, and decreased cost.

With the incremental improvement in viral load suppression and the expanding antiretroviral armamentarium, opportunistic infections and HIV-related mortality have significantly declined precluding their use as measures of efficacy as impossibly large sample sizes would be required to detect statistically and clinically important differences. ${ }^{6}$ This leaves targeted surrogate outcomes as the markers of efficacy and, given the number of randomized clinical trials assessing antiretrovirals, it is necessary to standardize these to allow for appropriate comparisons. As such, the US Food and Drug Administration (FDA) Snapshot algorithm is commonly used to evaluate for effectiveness along with a comparable mechanism for classifying adverse events. ${ }^{7,8}$

INSTIs, the newest class of antiretrovirals, act by preventing HIV DNA from incorporating into the host T-lymphocyte genome, limiting virus propagation. Raltegravir, requiring twice-daily dosing, and elvitegravir, requiring concurrent cobicistat, were the first to be approved. ${ }^{9-13}$ Dolutegravir, the most recent INSTI, can be taken once
Toronto General Hospital, Eaton Wing 13th Floor, Room 214, 200 Elizabeth Street, Toronto, ON M5G 2C4, Canada Emailsharon.walmsley@uhn.ca 
daily and has been co-formulated into a single-tablet regimen (STR) with abacavir and lamivudine that was recently granted FDA approval. ${ }^{14}$ Currently, dolutegravir, with either abacavir-lamivudine or tenofovir-emtricitabine, is included among the preferred initial antiretroviral regimens. ${ }^{1,15}$

The purpose of this review is to summarize the evidence pertaining to dolutegravir, focusing on the rationale for combination therapy.

\section{Pharmacology}

Dolutegravir acts by impairing the function of the HIV integrase-DNA complex to which it was chemically synthesized to bind. ${ }^{16}$ It is rapidly absorbed, achieving maximal blood concentration hours after ingestion and, with a terminal halflife of 12 hours, requires once-daily administration without pharmacological enhancement. ${ }^{17-19}$ There is minimal urinary excretion as it is metabolized predominantly through hepatic glucuronidation by UDP-glucuronosyltransferase $1 \mathrm{~A} 1 .^{17,20}$ Given the low renal elimination, reduced renal function does not significantly alter the pharmacokinetics of dolutegravir. ${ }^{21}$ Whether this extends to patients receiving renal replacement therapy is unknown. Similarly, there is a dearth of evidence evaluating the impact of impaired hepatic function on the activity of dolutegravir. In a small comparison of those with Child-Pugh class B cirrhosis to healthy controls, the only difference was an increase in the unbound concentration of dolutegravir, the clinical significance of which is likely minimal as more than $99 \%$ remained in the active proteinbound form. ${ }^{22}$ Evidence of the wide distribution of dolutegravir comes from its detection in human colorectal tissue, cerebrospinal fluid, seminal fluid, cervicovaginal fluid, and vaginal tissue at concentrations above that expected to confer continued antiviral efficacy. ${ }^{23-25}$

Drug-drug interactions with dolutegravir are minimal as it has little ability to alter drug-metabolizing enzymes. There are no interactions or dose adjustments required when combined with the NRTI class, as bioequivalence was observed when dolutegravir and abacavir-lamivudine, administered separately, were compared to a co-formulated single tablet. ${ }^{26-28}$ Among the NNRTI class, both efavirenz and etravirine significantly lower dolutegravir levels and should be avoided unless etravirine is administered with ritonavir, which reverses this reduction. ${ }^{29,30}$ There is no interaction between rilpivirine and dolutegravir. ${ }^{31}$ The PIs darunavir, lopinavir, fosamprenavir, and atazanavir, irrespective of ritonavir coadministration, can be safely used with dolutegravir. ${ }^{32-34}$ Tipranavir, however, reduces the plasma concentration of dolutegravir and caution should be exercised with coadministration. ${ }^{30}$ Interactions between dolutegravir and cobicistat - currently being evaluated as an alternative pharmacokinetic enhancer to ritonavir - are unclear and require further investigation.

Coinfection with hepatitis $\mathrm{C}$ and tuberculosis frequently occur, and the lengthy treatment regimens consisting of multiple agents make interactions with antiretrovirals inevitable. While there are no interactions between dolutegravir and boceprevir or telaprevir, the explosion of new antiviral agents active against hepatitis $\mathrm{C}$ will require pharmacokinetic studies to establish the feasibility of concurrent administration. ${ }^{35}$ Given the mechanism of metabolism of dolutegravir and with no clinically significant interactions between it and grazoprevir with elbasvir, it is expected that concurrent use with the direct acting agents against hepatitis $\mathrm{C}$ should not impact drug levels, but clinical data are lacking. ${ }^{15,36}$ As for tuberculosis therapy, rifampin lowers the concentration of dolutegravir, which can be offset by increasing the frequency of dolutegravir (50 mg twice daily) or substituting rifabutin as no adjustments are required. ${ }^{37}$

Outside of antimicrobial agents, dolutegravir has few drug-drug interactions. There does not appear to be a significant interaction between dolutegravir and oral contraceptive pills or proton pump inhibitors. ${ }^{38,39}$ Antacids, however, can attenuate the effectiveness of dolutegravir, which should be taken 2 hours prior to or 6 hours following the ingestion of an antacid. ${ }^{39}$ Such a schedule should likewise be followed if dolutegravir is taken with cations such as iron and calcium, although these interactions can be avoided when ingested with a moderately fatty meal. ${ }^{40}$ Dolutegravir alters the pharmacokinetics of metformin, possibly enhancing gastrointestinal upset. ${ }^{41}$ In the absence of mineral supplements, dolutegravir can be taken with or without food. ${ }^{42}$

\section{Dolutegravir efficacy Antiretroviral-naïve patients}

Dose response studies determined $50 \mathrm{mg}$ of dolutegravir as the most efficacious, with similar side effects as lower daily doses. ${ }^{43,44}$ In a blinded study, SPRING-2, comparing raltegravir against dolutegravir with either abacavir-lamivudine or tenofovir-emtricitabine, once-daily dolutegravir was noninferior, with $88 \%$ and $85 \%$, respectively, achieving viral load suppression. ${ }^{45}$ This effect diminished slightly, but noninferiority persisted to 96 weeks. ${ }^{46}$ Failure to achieve virologic suppression was entirely due to discontinuation of dolutegravir for reasons other than the development of resistance, which was not observed. Against darunavirritonavir in the open-label FLAMINGO study, ${ }^{47}$ dolutegravir 
led to virologic suppression in $90 \%$ of patients at 48 weeks compared with $83 \%$ in the darunavir-ritonavir group, which was predominantly the result of discontinuation due to adverse events, but also some improvement in efficacy above 100,000 copies per milliliter. The open-label nature of FLAMINGO could have led to biases in discontinuation rates. ${ }^{47}$ Similar to SPRING-2, the effect waned slightly, but remained statistically significant at 96 weeks. ${ }^{48}$ In SINGLE, a randomized placebo-controlled study comparing dolutegravir with abacavir-lamivudine against tenofovir-emtricitabineefavirenz, viral load suppression occurred in $88 \%$ and $81 \%$ at 48 weeks, respectively. ${ }^{26}$ The superiority of dolutegravir with abacavir-lamivudine persisted at 144 weeks. ${ }^{49}$ The benefit was driven almost entirely by increased discontinuations due to adverse events associated with efavirenz. The unique aspect of SINGLE resides with controlling backbone agents as the aforementioned randomized trials entrusted backbone selection to study investigators. When all Phase III randomized trials were amalgamated, subgroup analysis did not find that patient age, backbone, or pretreatment viral load impacted effectiveness. ${ }^{50,51}$ Dolutegravir has been compared against PIs, NNRTIs, and INSTIs in treatment-naïve patients with consistent efficacy despite varying study populations (Table 1).

\section{Antiretroviral-experienced patients}

With the trend toward early initiation of antiretrovirals, the requirement for lifetime use, and myriad ways HIV escapes drug suppression, the proportion of treatment-experienced patients are naturally expected to rise. ${ }^{52}$ SAILING, a randomized trial of patients with resistance to at least two antiretroviral classes yet who were INSTI-naïve, compared raltegravir to dolutegravir with optimally constructed backbones. After 48 weeks, virologic suppression was observed in $71 \%$ in the dolutegravir cohort and $64 \%$ in the raltegravir cohort. The superiority of the dolutegravir regimen was observed irrespective of the background regimen, and resistance mutations were less likely to develop with dolutegravir. ${ }^{53}$ The biologic plausibility for the incremental benefit of dolutegravir over raltegravir is the slower dissociation from the HIV-1 integrase-DNA complex and the reduced interindividual pharmacokinetic variability. ${ }^{17,54}$

The VIKING trials assessed the utility of dolutegravir in populations with previous INSTI failure. In VIKING, patients with raltegravir resistance either by genotype analysis or treatment failure received dolutegravir once or twice daily for 10 days followed by optimization of the background regimen. After 24 weeks, almost twice as many subjects had an undetectable viral load in the twice-daily group (75\% to $41 \%) .{ }^{55}$
In VIKING-3, patients with historical or current evidence of resistance to either raltegravir or elvitegravir by genotype or phenotype testing were given dolutegravir twice daily for 7 days before optimizing the background regimen. After 24 weeks, 69\% achieved virologic suppression. ${ }^{56}$ VIKING-4 prospectively studied a heavily treatment-experienced cohort comparing a 7-day run-in period of dolutegravir or placebo followed by both groups receiving dolutegravir and an individually optimized background regimen. After 24 and 48 weeks, viral load suppression occurred in $47 \%$ and $40 \%$, respectively. ${ }^{57}$ In an open-label cohort of heavily treatment-experienced HIV2-infected patients, dolutegravir led to an undetectable viral load in $38 \% .^{58}$ Cumulatively, these studies support the use of twice-daily dolutegravir among those with raltegravir or elvitegravir failure (Table 2). Success with the sequential use of dolutegravir following INSTI failure is predicated on the presence of at least two active backbone agents and reduced development of INSTI resistance mutations. Thus, patients should stop a failing raltegravir- or elvitegravir-containing regimen as soon as possible to avoid the accumulation of mutations potentially compromising subsequent use of dolutegravir.

\section{STR with dolutegravir}

The bioequivalence of the co-formulated tablet leaves little doubt as to the potential efficacy of an STR containing dolutegravir. ${ }^{28}$ Given that there have been no published studies of dolutegravir as an STR and the consistent underrepresentation of women in the aforementioned trials, the Antiretroviral Therapy in Naïve Women (ARIA) trial was conducted, and the results are forthcoming. ARIA will compare the dolutegravir-abacavir-lamivudine STR against atazanavir-ritonavir with tenofovir-emtricitabine in treatment-naïve women. ${ }^{59}$ Further studies will address the feasibility of switching to the dolutegravir-abacavirlamivudine STR from either an INSTI-free regimen or from nevirapine with abacavir-lamivudine..$^{60,61}$

\section{Safety of dolutegravir Adverse events}

Amalgamating the adverse event profiles accrued from the randomized controlled trials of dolutegravir provides a robust evidence base. The total incidence of adverse effects approaches $90 \%$, but this liberal estimate consists of predominantly mild reactions that largely remit with time and may not entirely be drug related. Common adverse events include headache, nausea, and diarrhea, but the proportion with severe reactions (grade III or IV) is $1 \% .^{62}$ In SINGLE, as compared to SPRING and SAILING, the 


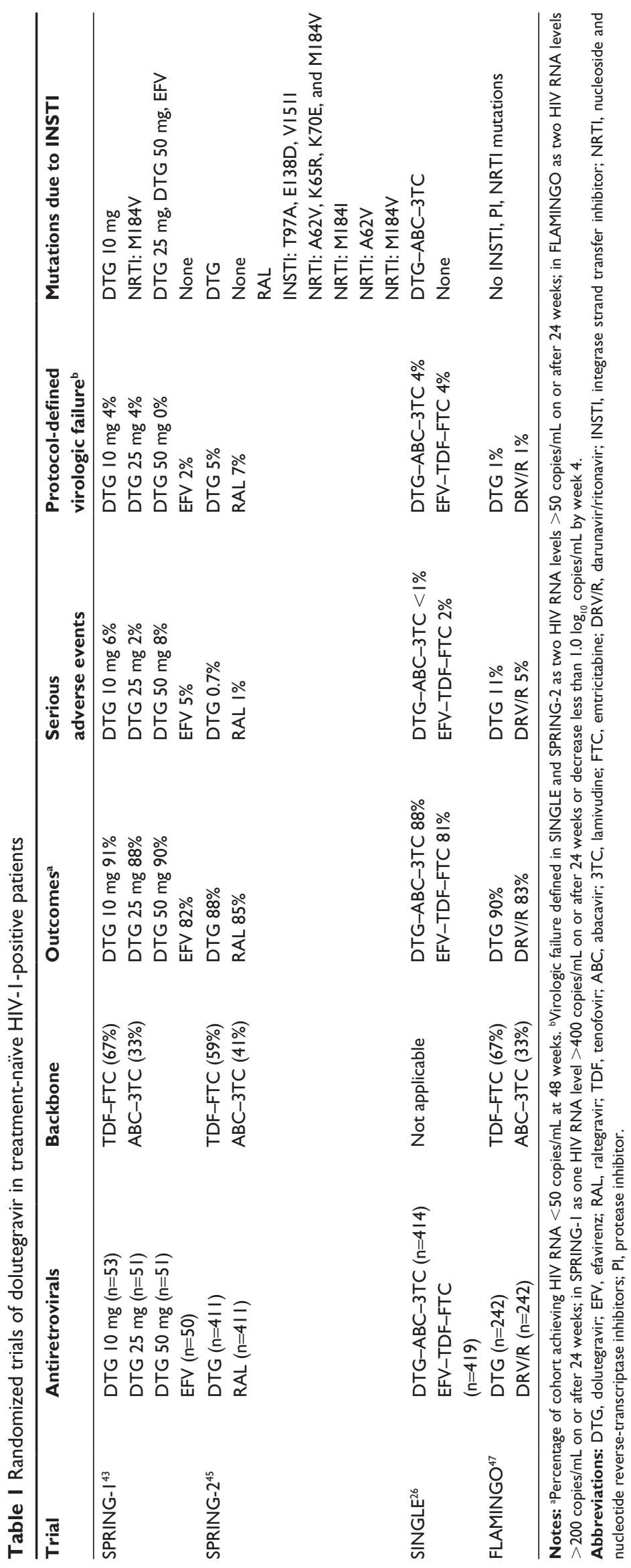


Table 2 Trials of dolutegravir in treatment-experienced HIV-I-positive patients

\begin{tabular}{|c|c|c|c|c|}
\hline Trial & Population & Antiretrovirals & Outcomes $^{a}$ & $\begin{array}{l}\text { Emergent DTG resistance } \\
\text { mutations }\end{array}$ \\
\hline SAILING ${ }^{53}$ & $\begin{array}{l}\text { Resistance to } \geq 1 \text { drug } \\
\text { in } \geq 2 \text { classes }\end{array}$ & $\begin{array}{l}\text { DTG }(n=354) \text { RAL } \\
(n=361)\end{array}$ & $\begin{array}{l}\text { At } 48 \text { weeks } \\
\text { DTG } 71 \% \\
\text { RAL } 64 \%\end{array}$ & $\begin{array}{l}\text { R263R (FC I.I2) } \\
\text { R263R (FC I.93) } \\
\text { R263R (FC I.I) } \\
\text { R263R (FC I.9) } \\
\text { EI38T/A + T97A (FC > max) } \\
\text { VI5II (FC 0.92) }\end{array}$ \\
\hline VIKING $55, c$ & $\begin{array}{l}\text { RAL resistance and } \geq 1 \\
\text { drug in } \geq 3 \text { classes }\end{array}$ & $\begin{array}{l}\text { DTG daily ( } n=27) \\
\text { DTG twice daily }(n=24)\end{array}$ & $\begin{array}{l}\text { At } 24 \text { weeks } \\
\text { DTG daily } 4 \text { I\% } \\
\text { DTG twice daily } 75 \%\end{array}$ & $\begin{array}{l}\text { L74I/M, EI38A (FC 38) } \\
\text { L74M/I, T97A, GI40S, QI48H (FC 68) } \\
\text { NI55H (FC 6.6) } \\
\text { NI55H (FC 8.4) } \\
\text { T97A, EI38K, NI55H (FC 93) } \\
\text { E92Q, T97A (FC 42) } \\
\text { EI38K, NI55H (FC 63) }\end{array}$ \\
\hline VIKING-3 ${ }^{56}$ & $\begin{array}{l}\text { RAL/ELV resistance and } \\
\geq 1 \text { drug in } \geq 3 \text { classes }\end{array}$ & DTG twice daily $(\mathrm{n}=183)$ & $\begin{array}{l}\text { At } 24 \text { weeks } \\
\text { DTG } 69 \%\end{array}$ & Not available \\
\hline VIKING-4 ${ }^{57}$ & $\begin{array}{l}\text { RAL/ELV resistance and } \\
\geq 1 \text { drug in } \geq 2 \text { classes }\end{array}$ & DTG twice daily $(n=30)$ & $\begin{array}{l}\text { At } 24 \text { weeks } \\
\text { DTG } 47 \%\end{array}$ & $\begin{array}{l}\text { L74L/M }^{b} \\
\text { T97A } \\
\text { T97A } \\
\text { T97A } \\
\text { EI38K } \\
\text { SI47G } \\
\text { NI55H }\end{array}$ \\
\hline
\end{tabular}

Notes: aPercentage of cohort achieving HIV RNA <50 copies/mL at specified dates. ' $\mathrm{FC}$ not provided. 'Adapted from Eron JJ, Clotet B, Durant J, et al; VIKING Study Group. Safety and efficacy of dolutegravir in treatment-experienced subjects with raltegravir-resistant HIV type I infection: 24-week results of the VIKING Study. J Infect Dis. 2013;207(5):740-748. ${ }^{55}$

Abbreviations: RAL, raltegravir; DTG, dolutegravir; ELV, elvitegravir; FC, fold change in phenotype resistance; max, maximum.

prevalence of insomnia was higher, which may be related to the specific study questionnaire that had not been employed in the previous trials. ${ }^{26}$ In a meta-analysis, there were significantly fewer adverse events with dolutegravir as compared to atazanavir-ritonavir, lopinavir-ritonavir, and efavirenz, while no differences between darunavirritonavir, elvitegravir-cobicistat, raltegravir, and rilpivirine were observed. ${ }^{63}$ Furthermore, adverse events ascribed to dolutegravir infrequently led to treatment cessation, occurring in less than $2 \%$, comparable to raltegravir and lower than efavirenz and PI-based regimens. ${ }^{63}$ When compared to raltegravir in treatment-experienced patients, there was no difference in the overall frequency of adverse events nor in the frequency of adverse events leading to drug discontinuation..$^{53}$ Dolutegravir has not been associated with an increase in cardiovascular risk. ${ }^{26,45,47}$ Further proof of the tolerability of dolutegravir is the similar side effect profile observed when given twice daily, even among those with advanced immunosuppression..$^{55,56}$

With respect to biochemical perturbations due to dolutegravir, the most consistently observed is creatinine elevation. This typically occurs within a week of initiation followed by a plateau at an average increase of $11 \mathrm{mmol} / \mathrm{L} .{ }^{62}$ This rise is mediated through inhibition of the renal transporter OCT-2, but the reduced creatinine secretion does not translate into a lower glomerular filtration rate. ${ }^{64}$ Elevations in transaminases occur in 5\%, are generally mild, and occur at a similar rate as with raltegravir, darunavir-ritonavir, and efavirenz. ${ }^{62}$ In the limited number of patients with hepatitis B or $\mathrm{C}$ coinfection, the incidence of transaminase elevation rises to $16 \%$, most likely reflecting immune reconstitution, and is lower than that observed with raltegravir and efavirenz, but higher than that with darunavir-ritonavir. ${ }^{62}$ Elevation in total cholesterol, low-density lipoprotein, and triglycerides observed with PIs is absent with dolutegravir. ${ }^{62}$ Creatine kinase elevations are common, largely asymptomatic, and mild, with only $5 \%$ being grade III or IV in severity. ${ }^{62}$ Hypersensitivity reactions were extremely uncommon, occurring in less than $1 \%$, and tend to occur shortly after treatment initiation. ${ }^{62}$

\section{Special populations}

There is a paucity of information regarding the use of dolutegravir in pediatric and pregnant populations. In animal studies, dolutegravir crosses the placenta, but this had no impact on fetal development in rats and rabbits despite 
exposure to supratherapeutic doses, resulting in an FDA class B classification. ${ }^{65}$ Ongoing clinical trials evaluating dolutegravir in treatment-experienced children and pregnant women will clarify the safety and efficacy of dolutegravir in these populations. ${ }^{66,67}$ In the interim, dolutegravir is not recommended in pregnancy unless alternative agents are unavailable.

\section{Resistance profile}

Another advantage of dolutegravir relates to the barrier to resistance. When analyzing those experiencing virologic failure while on dolutegravir as first-line therapy, no resistance mutations were discovered. ${ }^{68}$ This contrasts with the four of 281 patients who developed raltegravir resistance in STARTMRK at 5 years and one of 411 patients in SPRING-2 at 96 weeks. ${ }^{13,45}$ In comparison, at week 144, elvitegravirresistant virus was observed in nine of 348 patients and six of 353 patients in studies comparing it to efavirenz and atazanavir-ritonavir, respectively. ${ }^{69,70}$ It is unclear as to whether the resistance barrier to dolutegravir is similar to or surpasses that of PIs, as virologic failure due to resistance was not observed in FLAMINGO. ${ }^{47}$

Dolutegravir has induced mutations within the integrase enzyme, but these are infrequent and have minimal effect clinically. Dolutegravir can select for a R263K mutation that attenuates its activity, but not to an extent that allows for viral rebound. ${ }^{71}$ Continual dolutegravir selection pressure allows for the development of sequential mutations, generally in the same R263K pathway, but again these do not substantially impact antiviral activity and may in fact confer reduced HIV replication fitness. ${ }^{52,72-75}$

It is important to note that the randomized clinical trials evaluating dolutegravir test for resistance upon detection of viral rebound, which often differs from clinical practice, where patients can remain on failing regimens for longer before genotype analysis is undertaken. This allows for additional selection pressure and may serve to increase the incidence of dolutegravir resistance. Given that adherence may be less optimal outside the rigor of clinical trials, over time the increasing use of dolutegravir may result in the emergence of novel mutations. Recently, a patient with known N155H, S119R, and E157Q mutations who achieved suppression with dolutegravir experienced virologic rebound conferred by novel mutations, T97A and S147G ${ }^{76}$ This further confirms the importance of modifying a failing regimen urgently to avoid the accumulation of mutations that may compromise therapy. ${ }^{77}$

\section{Patient-reported outcomes}

Not captured in the randomized trials of dolutegravir are subjective measures of a patient's health - termed "patientreported outcomes". A number of assessment tools have been evaluated, principally among those receiving NNRTIor PI-based regimens, but none are sufficiently robust for widespread adoption. ${ }^{78}$ When these infrequently ascertained measures are assessed, as in SINGLE, dolutegravir is not inferior to tenofovir-emtricitabine-efavirenz. ${ }^{26}$

Maximizing adherence to antiretroviral treatment is vital and became even more important following the recognition that multiple antiretrovirals with varying mechanisms of action were required for continual HIV suppression. ${ }^{79,80}$ Strategies to improve adherence, including reducing pill burden to simplify regimens, should translate into improved quality of life. ${ }^{81}$ As an added benefit, co-formulated STRs, when compared to the component antivirals taken separately, may potentially reduce the development of resistance mutations ${ }^{82-86}$ Furthermore, an initial highly successful regimen obviates the need to switch therapy, which may result in experiencing new side effects that negatively impact quality of life. ${ }^{87,88}$ As the initial antiretroviral regimen predicts successful long-term virologic suppression, selecting the correct therapy is critically important. ${ }^{89}$

\section{Conclusion}

Dolutegravir, the newest INSTI, is an effective antiretroviral agent for both treatment-naïve and treatment-experienced patients infected with HIV. This is driven by effective virologic suppression, good tolerability, infrequent drug-drug interactions, and once-daily administration. Co-formulation of dolutegravir with abacavir-lamivudine maximizes adherence and should be considered among the initial options for the treatment-naïve. In the treatment-experienced, including those with INSTI resistance, dolutegravir remains effective when taken twice daily. Future studies will hopefully extend the effectiveness to populations underrepresented in current clinical trials. Perhaps the most novel aspect of dolutegravir is the high barrier to resistance, which may have important public health implications by reducing the development, and subsequent transmission, of resistance mutations.

\section{Disclosure}

Sharon L Walmsley has served on advisory boards, spoken at Continuing Medical Education-related events, and conducted clinical trials with Viiv, Bristol Meyers Squibb, Abbvie, Jannsen Pharmaceuticals, Gilead, and Merck and Co 
Incorporate. The authors report no other conflicts of interest in this work.

\section{References}

1. Günthard HF, Aberg JA, Eron JJ, et al; International Antiviral SocietyUSA Panel. Antiretroviral treatment of adult HIV infection: 2014 recommendations of the International Antiviral Society-USA Panel. JAMA. 2014;312(4):410-425.

2. Warnke D, Barreto J, Temesgen Z. Antiretroviral drugs. J Clin Pharmacol. 2007;47(12):1570-1579.

3. Gandhi M, Gandhi RT. Single-pill combination regimens for treatment of HIV-1 infection. N Engl J Med. 2014;371(3):248-259.

4. Samji H, Cescon A, Hogg RS, et al; the North American AIDS Cohort Collaboration on Research and Design (NA-ACCORD) of IeDEA. Closing the gap: increases in life expectancy among HIV-positive individuals in the United States and Canada. PLoS One. 2013;8(12): e81355.

5. Rodger AJ, Lodwick R, Schechter M, et al; INSIGHT SMART, ESPRIT Study Groups. Mortality in well controlled HIV in the continuous antiretroviral arms of the SMART and ESPRIT trials compared with the general population. AIDS. 2013;27(6):973-979.

6. Walensky RP, Paltiel AD, Losina E, et al. The survival benefits of AIDS treatment in the United States. J Infect Dis. 2006;194(1):11-19.

7. Qaqish R, van Wyk J, King MS. A comparison of the FDA TLOVR and FDA Snapshot algorithms based on studies evaluating once-daily vs twice daily lopinavir/ritonavir (LPV/r) regimens. J Int AIDS Soc. 2010;13(Suppl 4):P58.

8. Division of AIDS (DAIDS) Table for Grading the Severity of Adult and Pediatric Adverse Events, Version 2.0. Division of AIDS, National Institute of Allergy and Infectious Diseases, National Institutes of Health, US Department of Health and Human Services; 2014. Available from: http://rsc.tech-res.com/Document/safetyandpharmacovigilance/ DAIDS_AE_GRADING_TABLE_v2_NOV2014.pdf. Accessed March 7, 2015.

9. Sax PE, DeJesus E, Mills A, et al; GS-US-236-0102 study team. Co-formulated elvitegravir, cobicistat, emtricitabine, and tenofovir versus co-formulated efavirenz, emtricitabine, and tenofovir for initial treatment of HIV-1 infection: a randomised, double-blind, phase 3 trial, analysis of results after 48 weeks. Lancet. 2012;379(9835): 2439-2348.

10. DeJesus E, Rockstroh JK, Henry K, et al; GS-236-0103 Study Team. Co-formulated elvitegravir, cobicistat, emtricitabine, and tenofovir disoproxil fumarate versus ritonavir-boosted atazanavir plus co-formulated emtricitabine and tenofovir disoproxil fumarate for initial treatment of HIV-1 infection: a randomised, double-blind, phase 3, non-inferiority trial. Lancet. 2012;379(9835):2429-2438.

11. Zolopa A, Sax PE, DeJesus E, et al; GS-US-236-0102 Study Team. A randomized double-blind comparison of coformulated elvitegravir/ cobicistat/emtricitabine/tenofovir disoproxil fumarate versus efavirenz/ emtricitabine/tenofovir disoproxil fumarate for initial treatment of HIV-1 infection: analysis of week 96 results. J Acquir Immune Defic Syndr. 2013;63(1):96-100.

12. Lennox JL, DeJesus E, Lazzarin A, et al; STARTMRK investigators. Safety and efficacy of raltegravir-based versus efavirenz-based combination therapy in treatment-naive patients with HIV-1 infection: a multicentre, double-blind randomised controlled trial. Lancet. 2009; 374(9692):796-806.

13. Rockstroh JK, DeJesus E, Lennox JL, et al; STARTMRK Investigators. Durable efficacy and safety of raltegravir versus efavirenz when combined with tenofovir/emtricitabine in treatment-naive HIV-1-infected patients: final 5-year results from STARTMRK. J Acquir Immune Defic Syndr. 2013;63(1):77-85.

14. Gohil K. Pharmaceutical approval update. $P$ T. 2014;39(11): 746-772.
15. Panel on Antiretroviral Guidelines for Adults and Adolescents. Guidelines for the Use of Antiretroviral Agents in HIV-1-Infected Adults and Adolescents. Department of Health and Human Services; 2015. Available from: http://aidsinfo.nih.gov/ContentFiles/Adultand AdolescentGL.pdf. Accessed March 7, 2015.

16. Garvey EP, Johns BA, Gartland MJ, et al. The naphthyridinone GSK364735 is a novel, potent human immunodeficiency virus type 1 integrase inhibitor and antiretroviral. Antimicrob Agents Chemother. 2008;52(3):901-908.

17. Cottrell ML, Hadzic T, Kashuba AD. Clinical pharmacokinetic, pharmacodynamic and drug-interaction profile of the integrase inhibitor dolutegravir. Clin Pharmacokinet. 2013;52(11):981-994.

18. Min S, Song I, Borland J, et al. Pharmacokinetics and safety of S/GSK1349572, a next-generation HIV integrase inhibitor, in healthy volunteers. Antimicrob Agents Chemother. 2010;54(1):254-258.

19. Min S, Sloan L, DeJesus E, et al. Antiviral activity, safety, and pharmacokinetics/pharmacodynamics of dolutegravir as 10-day monotherapy in HIV-1-infected adults. AIDS. 2011;25(14):1737-1745.

20. Castellino S, Moss L, Wagner D, et al. Metabolism, excretion, and mass balance of the HIV-1 integrase inhibitor dolutegravir in humans. Antimicrob Agents Chemother. 2013;57(8):3536-3546.

21. Weller S, Borland J, Chen S, et al. Pharmacokinetics of dolutegravir in HIV-seronegative subjects with severe renal impairment. Eur J Clin Pharmacol. 2014;70(1):29-35.

22. Song IH, Borland J, Savina PM, et al. Pharmacokinetics of singledose dolutegravir in HIV-seronegative subjects with moderate hepatic impairment compared to healthy matched controls. Clin Pharmacol Drug Dev. 2013;2:342-348.

23. Greener BN, Patterson KB, Prince HM, et al. Dolutegravir pharmacokinetics in the genital tract and colorectum of HIV-negative men after single and multiple dosing. J Acquir Immune Defic Syndr. 2013;64(1):39-44.

24. Adams JL, Patterson KB, Prince HM, et al. Single and multiple dose pharmacokinetics of dolutegravir in the genital tract of HIV-negative women. Antivir Ther. 2013;18(8):1005-1013.

25. Letendre SL, Mills AM, Tashima KT, et al; extended ING116070 study team. ING116070: a study of the pharmacokinetics and antiviral activity of dolutegravir in cerebrospinal fluid in HIV-1-infected, antiretroviral therapy-naive subjects. Clin Infect Dis. 2014;59(7): 1032-1037.

26. Walmsley SL, Antela A, Clumeck N, et al; SINGLE Investigators. Dolutegravir plus abacavir-lamivudine for the treatment of HIV-1 infection. N Engl J Med. 2013;369(19):1807-1818.

27. Song I, Min SS, Borland J, et al. Lack of interaction between the HIV integrase inhibitor S/GSK1349572 and tenofovir in healthy subjects. J Acquir Immune Defic Syndr. 2010;55(3):365-367.

28. Weller S, Chen S, Borland J, Savina P, Wynne B, Piscitelli SC. Bioequivalence of a dolutegravir, abacavir, and lamivudine fixed-dose combination tablet and the effect of food. J Acquir Immune Defic Syndr. 2014; 66(4):393-398.

29. Song I, Borland J, Min S, et al. Effects of etravirine alone and with ritonavir-boosted protease inhibitors on the pharmacokinetics of dolutegravir. Antimicrob Agents Chemother. 2011;55(7):3517-3521.

30. Song I, Borland J, Chen S, et al. Effects of enzyme inducers efavirenz and tipranavir/ritonavir on the pharmacokinetics of the HIV integrase inhibitor dolutegravir. Eur J Clin Pharmacol. 2014;70(10): 1173-1179.

31. Ford SL, Gould E, Chen S, et al. Lack of pharmacokinetic interaction between rilpivirine and integrase inhibitors dolutegravir and GSK1265744. Antimicrob Agents Chemother. 2013;57(11):5472-5477.

32. Song I, Min SS, Borland J, et al. The effect of lopinavir/ritonavir and darunavir/ritonavir on the HIV integrase inhibitor S/GSK1349572 in healthy participants. J Clin Pharmacol. 2011;51(2):237-242.

33. Song I, Borland J, Chen S, et al. Effect of atazanavir and atazanavir/ritonavir on the pharmacokinetics of the next-generation HIV integrase inhibitor, S/GSK1349572. Br J Clin Pharmacol. 2011;72(1):103-108. 
34. Song I, Borland J, Chen S, Peppercorn A, Wajima T, Piscitelli SC. Effect of fosamprenavir-ritonavir on the pharmacokinetics of dolutegravir in healthy subjects. Antimicrob Agents Chemother. 2014;58(11):6696-6700.

35. Johnson M, Borland J, Chen S, Savina P, Wynne B, Piscitelli S. Effects of boceprevir and telaprevir on the pharmacokinetics of dolutegravir. Br J Clin Pharmacol. 2014;78(5):1043-1049.

36. Yeh W, Feng HP, Guo Z, et al. Drug-drug interaction between HCV inhibitors grazoprevir/elbasvir with dolutegravir. Poster presented at: Annual Conference on Retroviruses and Opportunistic Infections; February 23-26, 2015; Seattle, WA.

37. Dooley KE, Sayre P, Borland J, et al. Safety, tolerability, and pharmacokinetics of the HIV integrase inhibitor dolutegravir given twice daily with rifampin or once daily with rifabutin: results of a phase 1 study among healthy subjects. J Acquir Immune Defic Syndr. 2013;62(1):21-27.

38. Tittle V, Bull L, Boffito M, Nwokolo N. Pharmacokinetic and pharmacodynamic drug interactions between antiretrovirals and oral contraceptives. Clin Pharmacokinet. 2015;54(1):23-34.

39. Patel P, Song I, Borland J, et al. Pharmacokinetics of the HIV integrase inhibitor S/GSK1349572 co-administered with acid-reducing agents and multivitamins in healthy volunteers. $J$ Antimicrob Chemother. 2011;66(7):1567-1572.

40. Song I, Borland J, Arya N, Wynne B, Piscitelli S. Pharmacokinetics of dolutegravir when administered with mineral supplements in healthy adult subjects. J Clin Pharmacol. 2015;55(5):490-496.

41. Zong J, Borland J, Jerva F, Wynne B, Choukour M, Song I. The effect of dolutegravir on the pharmacokinetics of metformin in healthy subjects. J Int AIDS Soc. 2014;17(4 Suppl 3):19584.

42. Song I, Borland J, Chen S, et al. Effect of food on the pharmacokinetics of the integrase inhibitor dolutegravir. Antimicrob Agents Chemother. 2012;56(3):1627-1629.

43. van Lunzen J, Maggiolo F, Arribas JR, et al. Once daily dolutegravir (S/GSK1349572) in combination therapy in antiretroviral-naive adults with HIV: planned interim 48-week results from SPRING-1, a doseranging, randomised, phase $2 \mathrm{~b}$ trial. Lancet Infect Dis. 2012;12(2): 111-118.

44. Stellbrink HJ, Reynes J, Lazzarin A, et al; SPRING-1 Team. Dolutegravir in antiretroviral-naive adults with HIV-1: 96-week results from a randomized dose-ranging study. AIDS. 2013;27(11):1771-1778.

45. Raffi F, Rachlis A, Stellbring HJ, et al; SPRING-2 Study Group. Once daily dolutegravir versus raltegravir in antiretroviral-naive adults with HIV-1 infection: 48 week results from the randomised, doubleblind, non-inferiority SPRING-2 study. Lancet. 2013;381(9868): 735-743.

46. Raffi F, Jaeger H, Quiros-Roldan E, et al; extended SPRING-2 Study Group. Once-daily dolutegravir versus twice-daily raltegravir in antiretroviral-naive adults with HIV-1 infection (SPRING-2 study): 96 week results from a randomised, double-blind, non-inferiority trial. Lancet Infect Dis. 2013;13(11):927-935.

47. Clotet B, Feinberg J, van Lunzen J, et al; ING114915 Study Team. Once-daily dolutegravir versus darunavir plus ritonavir in antiretroviralnaïve adults with HIV-1 infection (FLAMINGO): 48 week results from randomised open-label phase 3b study. Lancet. 2014;383(9936): 2222-2231.

48. Molina JM, Clotet B, van Lunzen J, et al. Once-daily dolutegravir is superior to once-daily darunavir/ritonavir in treatment-naïve HIV-1positive individuals: 96 week results from FLAMINGO. $J$ Int AIDS Soc. 2014;17(4 Suppl 3):19490.

49. Pappa K, Baumgarten A, Felizarta F, et al. Dolutegravir (DTG) + abacavir/lamivudine once daily superior to tenofovir/emtricitabine/ efavirenz in treatment naïve HIV subjects: 144-week results from SINGLE (ING114467). Poster presented at: Annual Interscience Conference of Antimicrobial Agents and Chemotherapy; September 5-9, 2014; Washington, DC.

50. Raffi F, Rachlis A, Brinson C, et al. Dolutegravir efficacy at 48 weeks in key subgroups of treatment-naive HIV-infected individuals in three randomized trials. AIDS. 2015;29(2):167-174.
51. Granier C, Givens N, Cuffe R, et al. Consistency of dolutegravir treatment difference in HIV+ treatment naives at week 96. Poster presented at: Annual Conference on Retroviruses and Opportunistic Infections; February 23-26, 2015; Seattle, WA.

52. Wainberg MA, Zaharatos GJ, Brenner BG. Development of antiretroviral drug resistance. $N$ Eng J Med. 2011;365(7):637-646.

53. Cahn P, Pozniak AL, Mingrone H, et al; extended SAILING Study Team. Dolutegravir versus raltegravir in antiretroviral-experienced, integrase-inhibitor-naive adults with HIV: week 48 results from the randomised, double-blind, non-inferiority SAILING study. Lancet. 2013;382(9893):700-708.

54. Hightower KE, Wang R, Deanda F, et al. Dolutegravir (S/GSK1349572) exhibits significantly slower dissociation than raltegravir and elvitegravir from wild-type and integrase inhibitor-resistant HIV-1 integrase-DNA complexes. Antimicrob Agents Chemother. 2011;55(10):4552-4559.

55. Eron JJ, Clotet B, Durant J, et al; VIKING Study Group. Safety and efficacy of dolutegravir in treatment-experienced subjects with raltegravir-resistant HIV type 1 infection: 24-week results of the VIKING Study. J Infect Dis. 2013;207(5):740-748.

56. Castagna A, Maggiolo F, Penco G, et al; VIKING-3 Study Group. Dolutegravir in antiretroviral-experienced patients with raltegravirand/or elvitegravir-resistant HIV-1: 24-week results of the phase III VIKING-3 study. J Infect Dis. 2014;210(3):354-362.

57. Akil B, Blick G, Hagins DP, et al; the VIKING-4 study team. Dolutegravir versus placebo in subjects harbouring HIV-1 with integrase inhibitor resistance associated substitutions: 48-week results from VIKING-4, a randomized study. Antivir Ther. Epub 2014 Oct 16.

58. Descamps D, Peytavin G, Visseaux B, et al. Dolutegravir in HIV-2-infected patients with resistant virus to first-line integrase inhibitors from the French Named Patient Program. Clin Infec Dis. 2015;60(10):1521-1527.

59. ViiV Healthcare. A study to determine safety and efficacy of dolutegravir/ abacavir/lamivudine (DTG/ABC/3TC) in human immunodeficiency virus (HIV)-1 infected antiretroviral therapy (ART) naïve women (ARIA). Available from: https:/clinicaltrials.gov/ct2/show/NCT01910402. NLM identifier: NCT01910402. Accessed March 7, 2015.

60. ViiV Healthcare. A Phase IIIb study of the safety, efficacy, and tolerability of switching to a fixed-dose combination of abacavir/dolutegravir/ lamivudine from current antiretroviral regimen. Available from: https:// www.clinicaltrials.gov/ct2/show/NCT02131025. NLM identifier: NCT02131025. Accessed March 7, 2015.

61. Nantes University Hospital. Multicentric open-label study of switch from abacavir/lamivudine fixed dose combination plus nevirapine to abacavir/ lamivudine/dolutegravir in virologically suppressed HIV-1 infected adults (SWAD). Available from: https://clinicaltrials.gov/ct2/show/NCT02067767. NLM identifier: NCT02067767. Accessed March 7, 2015.

62. Curtis L, Nichols G, Stainsby C, et al. Dolutegravir: clinical and laboratory safety in integrase inhibitor-naive patients. HIV Clin Trials. 2014;15(5):199-208.

63. Patel DA, Snedecor SJ, Tang WY, et al. 48-week efficacy and safety of dolutegravir relative to commonly used third agents in treatment-naive HIV-1-infected patients: a systematic review and network meta-analysis. PLoS One. 2014;9(9):e105653.

64. Koteff J, Borland J, Chen S, et al. A phase 1 study to evaluate the effect of dolutegravir on renal function via measurement of iohexol and paraamminohippurate clearance in healthy subjects. Br J Clin Pharmacol. 2013;75(4):990-996.

65. TIVICAY ${ }^{\circledR}$ (dolutegravir) [package insert]. Research Triangle Park, NC: GlaxoSmithKline; 2014.

66. National Institute of Allergy and Infectious Diseases (NIAID). Safety of and immune response to dolutegravir (GSK1349572) in HIV-1 infected infants, children, and adolescents. Available from: https:/clinicaltrials.gov/ct2/show/NCT01302847. NLM identifier: NCT01302847. Accessed March 7, 2015.

67. University of Liverpool. Safety and pharmacokinetics of dolutegravir in pregnant HIV mothers and their neonates: a pilot study (DolPHIN1). Available from: https://clinicaltrials.gov/ct2/show/NCT02245022. NLM identifier: NCT02245022. Accessed March 7, 2015. 
68. Mesplède T, Wainberg MA. Is resistance to dolutegravir possible when this drug is used in first-line therapy? Viruses. 2014;6(9):3377-3385.

69. Kulkarni R, Abram ME, McColl DJ, et al. Week 144 resistance analysis of elvitegravir/cobicistat/emtricitabine/tenofovir DF versus atazanavir+ ritonavir+emtricitabine/tenofovir DF in antiretroviral-naïve patients. HIV Clin Trials. 2014;15(5):218-230.

70. White KL, Kulkarni R, McColl DJ, et al. Week 144 resistance analysis of elvitegravir/cobicistat/emtricitabine/tenofovir DF versus efavirenz/ emtricitabine/tenofovir DF in antiretroviral-naive patients. Antvir Ther. Epub 2014 Oct 16.

71. Quashie PK, Mesplède T, Han YS, et al. Characterization of the R263K mutation in HIV-1 integrase that confers low-level resistance to second-generation integrase strand inhibitor dolutegravir. J Virol. 2012;86(5):2696-2705.

72. Wares M, Mesplède T, Quashie PK, Osman N, Han Y, Wainberg MA. The M50I polymorphic substitution in association with the R236K mutation in HIV-1 subtype B integrase increases drug resistance but does not restore viral replicative fitness. Retrovirology. 2014;11:7.

73. Mesplède T, Osman N, Wares M, et al. Addition of E138K to R263K in HIV integrase increases resistance to dolutegravir, but fails to restore activity of the HIV integrase enzyme and viral replication capacity. J Antimicrob Chemother. 2014;69(10):2733-2740.

74. Quashie PK, Mesplède T, Han YS, et al. Biochemical analysis of the role of G118R-linked dolutegravir drug resistance substitutions in HIV-1 integrase. Antimicrob Agents Chemother. 2013;57(12): 6223-6235.

75. Mesplède T, Quashie PK, Osman N, et al. Viral fitness cost prevents HIV-1 from evading dolutegravir drug pressure. Retrovirology. 2013;10:22.

76. Carganico A, Dupke S, Ehret R, et al. New dolutegravir resistance pattern identified in a patient failing antiretroviral therapy. J Int AIDS Soc. 2014;17(4 Suppl 3):19749.

77. Cavalcanti Jde S, Ferreira JL, Guimarães PM, Vidal JE, Brigido LF. High frequency of dolutegravir resistance in patients failing a raltegravircontaining salvage regimen. J Antimicrob Chemother. 2015;70(3): 926-929.

78. Simpson KN, Hanson KA, Harding G, et al. Patient reported outcome instruments used in clinical trials of HIV-infected adults on NNRTIbased therapy: a 10-year review. Health Qual Life Outcomes. 2013; $11: 164$.

79. Ammassari A, Trotta MP, Shalev N, Marconi P, Antinori A. Beyond virologic suppression: the role of adherence in the late HAART era. Antivir Ther. 2012;17(5):785-792.
80. Staszewski S, Miller V, Rehmet S, et al. Virological and immunological analysis of a triple combination pilot study with loviride, lamivudine and zidovudine in HIV-1-infected patients. AIDS. 1996;10(5):F1-F7.

81. Thompson MA, Mugavero MJ, Amico KR, et al. Guidelines for improving entry into and retention in care and antiretroviral adherence for persons with HIV: evidence-based recommendations from an International Association of Physicians in AIDS Care panel. Ann Intern Med. 2012;156(11):817-833.

82. Bangsberg DR, Ragland K, Monk A, Deeks SG. A single tablet regimen is associated with higher adherence and viral suppression than multiple tablet regimens in HIV+ homeless and marginally housed people. AIDS. 2010;24(18):2835-2840.

83. Blanco JL, Montaner JSG, Marconi VC, et al. Lower prevalence of drug resistance mutations at first-line virological failure to first-line therapy with atripla vs tenofovir+emtricitabine/lamivudine+efavirenz administered on a multiple tablet therapy. AIDS. 2014;28(17):2531-2539.

84. Engsig FN, Gerstoft J, Helleberg M, et al. Effectiveness of antiretroviral therapy in individuals who for economic reasons were switched from a once-daily single-tablet regimen to a triple-tablet regimen. J Acquir Immune Defic Syndr. 2014;66(4):407-413.

85. Hanna DB, Hessol NA, Golub ET, et al. Increase in single-tablet regimen use and associated improvements in adherence-related outcomes in HIVinfected women. J Acquir Immune Defic Syndr. 2014;65(5):587-596.

86. Sweet D, Song J, Zhong Y, Signorovitch J. Real-world medication persistence with single versus multiple tablet regimens for HIV-1 treatment. J Int AIDS Soc. 2014;17(4 Suppl 3):19537.

87. Abgrall S, Ingle SM, May MT, et al; Antiretroviral Therapy Cohort Collaboration (ART-CC) Durability of the first ART regimen and risk factors for modification, interruption or death in HIV-positive patients starting ART in Europe and North America 2002-2009. AIDS. 2013;27(5):803-813.

88. Carr A, Hoy J, Pozniak A. The ethics of switch/simplify in antiretroviral trials: non-inferior or just inferior. PLoS Med. 2012;9(7): e1001240

89. Lee FJ, Amin J, Carr A. Efficacy of initial antiretroviral therapy for HIV-1 infection in adults: a systematic review and meta-analysis of 114 studies with up to 144 weeks' follow-up. PLoS One. 2014;9(5): e97842.

\section{Publish your work in this journal}

Drug Design, Development and Therapy is an international, peerreviewed open-access journal that spans the spectrum of drug design and development through to clinical applications. Clinical outcomes, patient safety, and programs for the development and effective, safe, and sustained use of medicines are a feature of the journal, which

\section{Dovepress}

has also been accepted for indexing on PubMed Central. The manuscript management system is completely online and includes a very quick and fair peer-review system, which is all easy to use. Visit http://www.dovepress.com/testimonials.php to read real quotes from published authors. 